\title{
EDITORIAL
}

\section{Guideline for the Management of Patients with Atrial Fibrillation (AFib)}

The ACC, American Heart Association and the Heart Rhythm Society, in collaboration with the Society of Thoracic Surgery, have released a new 2014 Guideline for the Management of Patients With Atrial Fibrillation (AFib) that incorporates new and existing knowledge derived from published clinical trials, basic science, and comprehensive review articles, along with evolving treatment strategies and new drugs.

The guideline, which supersedes the "2006 ACC/AHA/ ESC Guideline for the Management of Patients With Atrial Fibrillation" and two subsequent focused updates from 2011, contains the most updated consensus of clinicians with broad expertise related to AFib and its treatment, including adult cardiology, electrophysiology, cardiothoracic surgery, and heart failure.

According to Writing Committee Chair Craig T. January, $\mathrm{MD}, \mathrm{PhD}$, the document contains four new significant recommendations. Specifically, the guideline calls for increased use of radio frequency ablation in the treatment of non-valvular atrial AFib, the addition of three new anticoagulants to treatment options, a diminished role in the use of aspirin, and a more comprehensive thromboembolic risk calculator called the CHA2DS2VASc calculator.

In the previous guideline, treatment protocols called for clinicians to prescribe aspirin to patients with AFib who had a low stroke risk, said January. However, he noted that data showing that aspirin decreases stroke risk "is weak." Previous treatment protocols also only recommended warfarin (Coumadin) as an anticoagulant for use in treating non-valvular AFib. The new guideline now includes recommendations for using dabigatran, rivaroxaban and apixaban - three new anticoagulants that have entered the marketplace within the last two years. The guideline also calls for a greater understanding of rate verses rhythm control treatment strategies in managing AFib.

Given the increasing incidence of AFib in an aging population, the connection of AFib to increased hospitalization, and the close association of AFib with other forms of cardiovascular disease, including hypertension, coronary artery disease and heart failure,
January notes that this new guideline is important for physicians and other members of the cardiovascular care team. To that end, he said the Writing Committee worked diligently to ensure that the data, tables, and figures were clear and easy to use. "The goal was to have a document that is useful to the practicing physician and makes sense," he said.

Moving forward, the guideline calls for additional studies to better inform clinicians as to the risks and benefits of therapeutic options for an individual patient, as well as continued research into the mechanisms that initiate and sustain AFib. Other recommendations include the need for new pharmacologic therapies, including antiarrhythmic drugs that have atrial selectivity and drugs that target fibrosis, and continued research into new anticoagulants. "Great promise lies in prevention," the document notes. "Future strategies for reversing the growing epidemic of AFib will come from basic science and genetic, epidemiologic, and clinical studies.”

The following are 10 points to remember about the 2014 guideline for the management of patients with nonvalvular atrial fibrillation (AF):

1. In assessing risk of stroke in a patient with nonvalvular $\mathrm{AF}$, the writing committee recommends (Class I) the usage of the $\mathrm{CHA}_{2} \mathrm{DS}_{2}$-VASc $(\mathrm{C}=$ congestive heart failure; $\mathrm{H}=$ hypertension; $\mathrm{A}_{2}=$ age $\geq 75$ years [doubled]; $\mathrm{D}=$ diabetes mellitus; $\mathrm{S}_{2}=$ stroke, transient ischemic attack, or thromboembolism [doubled]; $\mathrm{V}=$ vascular disease; $\mathrm{A}=$ age 65-74 years; $\mathrm{Sc}=$ sex category, i.e., female gender) score, as opposed to the $\mathrm{CHADS}_{2}$ score.

2. For nonvalvular AF patients with a history of stroke or transient ischemic attack, or a $\mathrm{CHA}_{2} \mathrm{DS}_{2}$-VASc score $\geq 2$, oral anticoagulation is recommended (Class I). Options for oral anticoagulation include warfarin, dabigatran, rivaroxaban, and apixaban.

3. For patients with nonvalvular $\mathrm{AF}$ and a $\mathrm{CHA}_{2} \mathrm{DS}_{2}-$ VASc score of 0 , it is reasonable to omit antithrombotic therapy (Class IIa).

4. The following options may be considered with a patient with nonvalvular AF and a $\mathrm{CHA}_{2} \mathrm{DS}_{2}$-VASc 
score of 1: no antithrombotic therapy, oral anticoagulation, or aspirin (Class IIb).

5. None of the novel oral anticoagulants (dabigatran, rivaroxaban, or apixaban) are recommended to be used in patients with AF and a mechanical or bioprosthetic heart valve (Class III harm).

6. As in the earlier guidelines, the committee recommends against the use of certain antiarrhythmic medications (flecainide, propafenone, dofetilide, and sotalol) in patients with severe left ventricular hypertrophy (LVH). In the current guidelines, severe $\mathrm{LVH}$ is now defined as wall thickness exceeding $1.5 \mathrm{~cm}$.

7. Oral anticoagulation should be prescribed to patients with hypertrophic cardiomyopathy and AF irrespective of the $\mathrm{CHA}_{2} \mathrm{DS}_{2}$-VASc score (Class I).

8. A randomized trial suggested that a lenient $(<110$ bpm) rate control strategy was as effective as a strict strategy $(<80 \mathrm{bpm})$ in patients with persistent/ permanent AF. However, the writing committee still advocates for the latter (Class IIa), as the results of this single trial were not thought to be definitive.

9. Catheter ablation is useful in patients with symptomatic, paroxysmal AF who have not responded to or tolerated antiarrhythmic medications (Class I).

10. Catheter ablation is also reasonable in selected patients with symptomatic, paroxysmal AF prior to a trial of medical therapy, provided that it can be performed at an experienced center (Class IIa).

\section{Dr. Harisul Hoque}

Associate Professor of Cardiology \&

Co-ordinator Heart Failure Clinic

Bangabandhu Sheikh Mujib Medical University (BSMMU), Dhaka.

\section{Reference:}

2014 AHA/ACC/HRS Atrial Fibrillation Guideline, January, CT et al. Journal of the American College of Cardiology PII: S07351097(14)01739-2, DOI: 10.1016/j.jacc.2014.03.021. 